of treatment and annually thereafter. Our aim was to determine if patients taking Orkambi experienced a rise in their liver enzymes during treatment.

Methods This study was conducted at the University Hospital Limerick, Ireland Paediatric CF unit. All 39 homozygous F508del patients on Orkambi were included. Lab results were collected for the following variables for each patient: AST, ALT, ALP, GGT and bilirubin at set time intervals Pre-Orkambi, and three, six, nine and 12 months on treatment. The Pre-Orkambi levels were compared with the three month levels.

Results We determined that overall patients on Orkambi did not have a rise in liver enzymes. Unexpectedly, patients had a statistically significant decrease in their ALT (p-value 0.016), ALP (p-value 0.001), GGT (p-value 0.002) and Bilirubin (pvalue 0.002 ). AST levels saw no significant change (p-value 0.76). Two patients had a rise in AST levels during Orkambi treatment greater than three times ULN, however, these levels then decreased with continued Orkambi use. None of our patients had any liver tests greater than eight times the ULN, or five times ULN during treatment, and none had ALT, ALP or Total Bilirubin levels greater than three times ULN during treatment. None of our patients had to stop taking Orkambi due to adverse events related to liver tests. Our findings are in contrast to those suggested by the original Orkambi trials data

Conclusion Our study demonstrated that patients taking Orkambi do not experience a rise in liver tests, in fact, unexpectedly, the values decrease.

\section{GP275 PEDIATRIC SPRAY-SOL: A NEW NEBULIZATION DEVICE SPECIFIC FOR UPPER RESPIRATORY TRACT INFECTIONS}

${ }^{1}$ Antonio Moffa, ${ }^{2}$ Valentina Grimaldi*, ${ }^{3}$ Vittorio Rinaldi, ${ }^{3}$ Michele Antonio Lopez, ${ }^{3}$ Lorenzo Sabatino, ${ }^{3}$ Andrea Costantino, 'Michele Cassano, ${ }^{3}$ Manuele Casale. 'Department of Otolaryngology, University of Foggia, Foggia, Italy; ${ }^{2}$ General Practice Paediatrician, Pubblic Health (Asl RM2), Rome, Italy; ${ }^{3}$ Unit of Otolaryngology, UOS ORL TI, Campus BioMedico University, Rome, Italy

\subsection{6/archdischild-2019-epa.334}

Background Upper respiratory tract infections (URTI) are very common in the pediatric population due to their 'physiologically' immature immune system; yet, around $90 \%$ are viral only $10 \%$ of URTI are bacterial. Therefore, treatment should essentially be symptomatic to relieve the child's discomfort and symptoms. Topical, rather than system, treatment is preferable reducing systemic effects and increasing the topical efficacy. There are many different nasal devices used to treat URTI, but often they are not specifically targeted for this purpose.

Objective Our group developed a new nebulization device, called 'Pediatric spray-sol', target specificity, very speed, pocked-size and portable, mono-patient without sterilization problems with the ability to vary concentration and quantity of medication in delivery, to combine substances in a single treatment.

Materials and Methods The device can be used in combination with a common syringe (without needle), without need for electricity. The user operates the plunger of the syringe to push the liquid drug into the delivery device. Once inside, the substance is nebulized and, from there, delivered into the nose in aerosol form. The device includes a fluidodynamic path with an expansion chamber and an acceleration chamber arranged in sequence, which allows for the optimum delivery of fluid.

Results The features of Spray- Sol include: the ability to nebulize high viscosity substances similarly an aerosol, nebulize particles of average diameter between 10 and 20 microns, the very low administration times (10 seconds to $5 \mathrm{cc}$ of substance), the cost and the possibility of use for all the duration of the treatment, it is portable and doesn't need electricity. This nasal nebulizer provides a more extensive and intensive delivery of solution in the nasal cavities, especially the deeper and higher portions of nasal cavity. In particular, pediatric Spray-sol, seem to guarantee a homogeneous distribution of drug at the middle turbinate and ostiomeatal complex crucial region for the drainage and ventilation of the paranasal sinuses. Spray-Sol reaches more easily the nasopharynx subsite and it could be considered a useful device for adenoiditis and Eustachian tube dysfunction. Moreover, it can quickly and efficiently nebulize not only salin solutions but others substances like hyaluronic acid, ectoine and corticosteroids.

Conclusions Pediatric Spray-sol is a new nebulization device for URTI treatment: combining the comfort of a spray with the efficacy of an aerosol. Pediatric Spray-sol can be used to deliver any type of solution in the nose such as saline solutions, hyaluronic acid, ectoine and corticosteroids.

\section{GP276 AUDIT OF NEWBORN SCREENING CORK UNIVERSITY HOSPITAL2011-2019 - IMPACT OF GESTATIONAL AGE ON SWEAT TESTING}

Aoife Gallagher*, Katherine Hooley, Sean Costello, Susanna Felsenstein, David Mullane, Muireann NíChróinín. Cork University Hospital, Cork, Ireland

\subsection{6/archdischild-2019-epa.335}

Background A previous national audit looking at sweat testing in all age groups in 2011 found Cork University Hospital (CUH) had a high number of tests with quantity not sufficient (QNS) result. Since then there has been new equipment and staff training. International laboratory audit criteria examine failure rates for under 6 months which has recently been $<1 \%$ in CUH (acceptable $<5 \%$ ). However higher failure rates $(0-40 \%)$ for sweat testing have been noted internationally in newborn screening programmes, a more difficult cohort to successfully sweat test.

Aims Sweat tests performed in CUH from 2011-2018 as part of newborn screening in relation to number of patients with positive $(>60)$, negative $(<30)$, equivocal and QNS results were reviewed. The gestational age at birth and at time of testing and birth weight were examined as potential variables.

\section{Methods}

Ethical approval obtained Newborn screening records for all patients from July 2011 to December 2018 reviewed. Sweat testing data obtained from laboratory. Data analysed using SPSS and Chi-Square test.

Results 102 infants referred to Cork for sweat testing as part of newborn screening. Patients diagnosed by meconium ileus $(n=7)$, had sweat testing elsewhere $(n=1)$ or had antenatal diagnosis $(n=2)$ were not included $(N=10)$. On initial testing 30 patients had a QNS result (32.6\%). 7 had same day repeat testing which was sufficient, the remaining having sweat testing at a later date. Ultimately there were 23 with positive tests, 67 with negative tests, 2 equivocal tests. Gestational age and birth weight of the 30 patients with initial QNS result 